\title{
Games of life
}

\author{
David A. Faux,* Mayank Shah, and Christopher Knapp \\ Department of Physics, University of Surrey, Guildford, GU2 7XH, UK
}

(Dated: February 27, 2020)

\begin{abstract}
Cellular automata are widely used in undergraduate physics courses to educate students in elementary programming and for project work. Cellular automata are coded with simple rules yet provide a rich if well-trodden landscape for exploring aspects of physics such as diffusion and magnetism. Mathematical games, such as the minority game or the prisoner's dilemma, are also amenable to project work with the added dimension of applications in finance, econophysics and social physics. Conway's classical game of life is both a mathematical game and a cellular automaton. We exploit adaptations of Conway's game of life as an opportunity for undergraduate students to explore new territory within the safe haven of an easy-to-implement cellular automaton. Students may discover new "lifeforms" comprising collections of live, dead and part-live cells, explore the escalation of floating-point errors leading to chaos-like behavior, amongst many phenomena not observed in Conway's classical counterpart.
\end{abstract}




\section{INTRODUCTION}

John von Neumann and Oskar Morgenstern are credited with introducing game theory in $1944^{1,2}$ prompting applications in economics, business, and the sciences. Popular games include the so-called "minority game", the "prisoner's dilemma" and Conway's "game of life" ${ }^{3}$. The prisoner's dilemma is a cross-disciplinary decision-making mathematical game that is relevant to physics and with applications in behavioral ecology as demonstrated by Hauert and Szabó ${ }^{4}$. The minority game ${ }^{5}$ is a mathematical game in which a large group of players make binary decisions based on strategies that depend on previous majority decisions. The minority game models stock-trader behavior. In both these games, players are seeking to maximise their worth but in doing so make decisions that depend on previous decisions by others. But the most well-known mathematical game is Conway's classical game of life ${ }^{3}$ and it is the adaptation of this game that forms the focus of this article.

Many mathematical games are also examples of cellular automata. A cellular automaton comprises a grid of cells in which each cell represents an entity that depends on the system to be simulated through rules that dictate how the system evolves at each iteration. Diffusion, magnetism, thermodynamics are amongst many subject areas that can be investigated through cellular automata. Cellular automata form the basis of computational physics

projects at undergraduate level because the computer coding is generally straightforward and because a vast range of different physical phenomena can be modeled. Mathematical game-based cellular automata have been shown to be effective at educating students in the emergence of complexity ${ }^{6}$ and logical thinking ${ }^{7}$. Students can develop their programming skills, and take ownership of their project work to explore phenomena of their choosing.

There is much literature on the use of "games" in education in the context of video or computer games. This article is about mathematical games of which the prisoner's dilemma, the game of life and the minority game form but three examples. The value of mathematical games in education was recognised as long ago as 1990 by Osborn in his article entitled A future for modelling in science education where he makes specific mention of cellular automata and, in particular, Conway's game of life ${ }^{8}$. Game theory courses appear in some undergraduate mathematics programmes but the application of mathematical games through computer simulation is most commonly found in project work in physics.

Conway's game of life mimics the evolution of a society of organisms in which the success 
or demise of life is based on the local environment; if the number of live cells in the neighborhood is too high or too low the cell dies due to overcrowding or loneliness but flourishes if the surrounding liveness is optimum. Each cell in the classical game of life evolves according to Conway's evolutionary rules and the simulation may be executed until a stable system is reached. Conway's evolutionary rules are simple and yet, when applied simultaneously to each cell at each time step (called a generation), an extraordinary myriad of complex patterns of live and dead cells emerge ${ }^{9}$. Conway's game merits an entertaining wikipedia page containing many examples of complex behaviors ${ }^{10}$ and an application available through Google Play.

Conway's classical game of life yields multicellular dynamic lifeforms which appear to move, yet there is no movement of individual cells within the game. Each cell is static, either alive or dead, and merely switches between alive and dead according to a set of rules. Games of life are not a random walk nor diffusion models. Nonetheless multicellular lifeforms are created which may be still-lifes, dynamic and/or oscillatory. In this article, we refer to stable patterns as "lifeforms". A lifeform is a collection of cells which, when placed in an otherwise empty system, either do not change under the rules of the game (referred to as "still-lifes"), return to their original pattern after a finite number of generations ("oscillators"), or move and oscillate ("spaceships").

The quantisation of cellular automata was recognised as important in the development of quantum computation following the suggestion by Feynman in $1982^{11}$. The quantisation of mathematical games eventually followed, most notably the quantum prisoner's dilemma ${ }^{12}$. A quantisation of Conway's classic game was first suggested in 2002 by Flitney and Abbott ${ }^{13}$ who proposed that a cell could be represented as a qubit. An educational illustration of the qubit is provided by López-Incera and Dür ${ }^{14}$. But Flitney and Abbott only explored this quantum game for a single illustrative one-dimensional qubit before moving to the burgeoning research area of quantum computing in which collections of qubits exist in superposed states.

In the semi-quantum game of life (SQGOL) as proposed and developed by Flitney and Abbott $^{13,15,16}$, each cell exists as an independent qubit. A set of rules adapted from Conway's game determines how the qubit evolves at each time step dependent on the liveness of its neighborhood. The SQGOL is thus distinctive from quantum computing in which collections of qubits exist in superposition and different from the quantum random walk where the 
probability density of a single qubit spreads across cells as a function of time. The rules of the SQGOL has a parallel in quantum biology whereby a qubit may, for example, represent an exciton produced by a photon in a protein complex during photosynthesis ${ }^{17}$. The exciton interacts with its environment to influence the energy transfer dynamics ${ }^{18}$. General quantum aspects of life are discussed in the book by Abbott and co-authors ${ }^{19}$.

Flitney and Abbott $^{16}$ proposed the rules that govern the semi-quantum version of the game of life but previous explorations of the SQGOL is limited ${ }^{20-22}$. Bleh and co-workers ${ }^{20}$ used Hamiltonian methods with creation and annihilation operators and Bagarello and coworkers $^{21}$ explored a quantum adaptation of Conway's game whilst maintaining live or dead cells only. A variant described as a "quantum game of life" is due to Arrighi and co-workers which is summarised in a popular article ${ }^{22}$. This is a three-dimensional quantum cellular automata that focuses on applications in quantum computing. Each approach demonstrates complexity but is not sufficiently simple to implement in a large-scale cellular simulation suitable for access by undergraduates.

In this article, the rules of the SQGOL are presented. A simplified version of the SQGOL is then described and explored. The simplified game does not make use of the quantum superposition principle, or complementarity, and thus translates the game to a classical game labelled as a "semi-classical game of life" (SCGOL) to distinguish it from both the SQGOL and Conway's game. The straightforward escalation to the SQGOL is left as one of many suggested enhancements. We present results for the SCGOL for the first time together with ideas for development as project work. It is shown that there are vast unexplored territories within both the SQGOL and SCGOL that would provide exciting opportunities for undergraduates to embark on original project work.

\section{GAME OF LIFE RULES}

Conway's game of life is based on a two-dimensional (2D) cellular automaton where each cell in a 2D grid is either 0 (dead) or 1 (alive). The system evolves in discrete time steps according to a set of simple rules ${ }^{3}$ which depend only on the number of live cells contained in its eight surrounding cells, known as the Moore neighborhood ${ }^{23}$. The "liveness" of the neighborhood is an integer between 0 and 8. Conway's rules stipulate that a live cell will die at the next time step if $A \leq 1$ or $A \geq 4$ and survives otherwise. A dead cell remains 
dead unless $A=3$ in which case the cell becomes alive.

The SQGOL scheme operates on the same principles as Conway's game but each cell is now a qubit. There is no movement of qubits from one cell to another. Each qubit remains static, it simply changes its degree of liveness according to adapted evolutionary rules. The rules of the SQGOL form the basis of a simplified SCGOL described in Section II B.

\section{A. The rules of the semi-quantum game of life}

A quantisation of Conway's game of life was proposed in 2002 by Flitney and Abbott ${ }^{13}$. Each cell of a 2D grid is described by a normalised state $|\psi\rangle$ which is a superposition of alive $|1\rangle$ and dead $|0\rangle$ states such that

$$
|\psi\rangle=a|1\rangle+b|0\rangle=a\left(\begin{array}{l}
1 \\
0
\end{array}\right)+b\left(\begin{array}{l}
0 \\
1
\end{array}\right)=\left(\begin{array}{l}
a \\
b
\end{array}\right) .
$$

The $a$ and $b$ are constants which may be complex. The SQGOL evolves each cell according to a set of rules which depend on the liveness, $A$, of the Moore neighborhood defined as ${ }^{16}$

$$
A e^{i \phi}=\sum_{k=1}^{8} a_{k}
$$

where $a_{k}$ is the (complex) liveness of the $k^{\text {th }}$ cell in the Moore neighborhood and where $k$ runs from 1 to 8 . In simple terms, the neighborhood liveness $A$ and phase $\phi$ are found by summing the complex $a_{k}$ of the individual neighboring qubits.

The evolutionary rules are based on the operators of "birth" $(\hat{B})$, "death" $(\hat{D})$, and "survival" $(\hat{S})$ given by ${ }^{13}$,

$$
\hat{B}=\left(\begin{array}{cc}
1 & e^{i \phi} \\
0 & 0
\end{array}\right) \quad \hat{D}=\left(\begin{array}{cc}
0 & 0 \\
e^{i \phi} & 1
\end{array}\right) \quad \hat{S}=\left(\begin{array}{ll}
1 & 0 \\
0 & 1
\end{array}\right)
$$

The next generation is obtained using the operation

$$
\left(\begin{array}{l}
a^{\prime} \\
b^{\prime}
\end{array}\right)=\hat{G}\left(\begin{array}{l}
a \\
b
\end{array}\right)
$$

where $\hat{G}$ is provided in Table $\mathrm{I}^{13}$. The operation is not unitary (probability density is not 


\begin{tabular}{cc}
\hline \hline $\mathbf{A}$ & $\hat{G}$ \\
\hline $0 \leq A \leq 1$ & $\hat{D}$ \\
$1<A \leq 2$ & $(\sqrt{2}+1)(2-A) \hat{D}+(A-1) \hat{S}$ \\
$2<A \leq 3$ & $(\sqrt{2}+1)(3-A) \hat{S}+(A-2) \hat{B}$ \\
$3<A \leq 4$ & $(\sqrt{2}+1)(4-A) \hat{B}+(A-3) \hat{D}$ \\
$A \geq 4$ & $\hat{D}$ \\
\hline \hline
\end{tabular}

TABLE I. The $\hat{G}$ operator for different values of $A$ (from Flitney and Abbott ${ }^{13}$ ).

conserved) and so the new state requires normalisation so that $\left|a^{\prime}\right|^{2}+\left|b^{\prime}\right|^{2}=1$. A cell is therefore a two-level system, or qubit $|\psi\rangle$, which interacts with its immediate environment comprising the eight cells of the Moore neighborhood to change the state to a new state $\left|\psi^{\prime}\right\rangle$. The extraordinary complexity of the SQGOL, as for Conway's classical game, arises because each cell simultaneously interacts with and is influenced by its neighborhood at each generation.

\section{B. Implementation of a semi-classical game of life}

The implementation of a modified game is presented that restricts the liveness and deadness of each cell, $a$ and $b$ respectively, to be real positive numbers. This leads to $\phi=0$ in Eqs. 2 and 3. The simplification of the rules employed makes the game equivalent to a classical superposition of states simulation as described by Flitney and Abbott ${ }^{13}$ and loses quantum effects such as interference, but acts as an excellent starting point for computations. We refer to this game as the semi-classical game of life, or SCGOL. The SCGOL allows "part-live" cells, that is cells with liveness represented by a number between 0 and 1 , in contrast to Conway's game that only allows integers 0 and 1 . We show that the SCGOL is rich with new phenomena and provides numerous avenues for investigation as project work including simple modification to the SQGOL.

In the SCGOL, the probability of finding a specific cell $i$ to be live if a liveness measurement is made is $a_{i}^{2}$. The system population is therefore represented as a probability density $a^{2}$, or population density, and the rules dictate how the liveness $a_{i}$ of each cell $i$, hence the population density, evolves in response to its environment. The game can be implemented straightforwardly by executing the following computational steps using any 
standard programming language.

(i) Set up five $100 \times 100$ matrices representing a universe of 10,000 cells. The first matrix contains the liveness $a$ of each cell, the second contains the deadness $b$, the third contains the liveness of the Moore neighborhood $A$ to each cell. The fourth and fifth matrices are initially empty but will contain the values of $a$ and $b$ at the next generation.

(ii) Initiate the system by assigning a fraction $f$ of cells in the first matrix to a liveness $a$ chosen as a random number uniformly distributed between 0 and 1 . The remaining cells have $a=0$. In the SCGOL, $a$ is real. The choice for $f$ should not be too small (otherwise the system will die out).

(iii) Set up the matrix for $b$. The value of $b$ for each cell is obtained by normalisation, that is $b=\sqrt{1-a^{2}}$.

(iv) The third matrix contains the Moore neighborhood $A$ for each cell calculated using Eq. 2. This step requires the application of periodic boundary conditions and thought will be required as to an efficient means of implementation.

(v) The SQGOL rules presented in section II A with $\phi=0$ are applied for each cell. First obtain the $\hat{G}$ operator using Table I for each cell in turn and then calculate the new values of liveness and deadness for that cell, $a^{\prime}$ and $b^{\prime}$, which are entered into the fourth and fifth matrices respectively.

(vi) Replace the original $a$ matrix by the $a^{\prime}$ and $b^{\prime}$ matrices divided by the normalisation constant. That is, $a=a^{\prime} / \sqrt{a^{\prime 2}+b^{\prime 2}}$. Produce the new matrix $b$.

(vii) At each generation, write key information to file for post processing. As a minimum, this should include the total system liveness (the sum of all the values of $a$ for the entire system) but could include a liveness matrix for the purpose of preparing a video.

(ix) Repeat processes (iv)-(vii) for a chosen number of generations. 


\section{RESULTS AND IDEAS}

In this section, we present some results from the SCGOL and make suggestions as to how the ideas may be developed for undergraduate project work. Even the SCGOL yields results substantially different to Conway's classical game and merely scrapes the surface of new game territory.

\section{A. Life, death and equilibrium}

The elementary simulations set out in Sec. II B will yield a dynamic equilibrium of liveness comprising dead, live and "part-live" cells as shown in Fig. 1. We refer to the liveness density as the "liveness cloud". The evolution of the mean system cell liveness $\langle a\rangle$ is presented in Fig. 2 for initial densities $f=0.2$ and $f=0.8$. The mean cell liveness at equilibrium is $<a>=0.3480 \pm 0.0001$ and the distribution of mean liveness is Gaussian with a standard deviation 0.0071. The same mean cell liveness and standard deviation at equilibrium are reached regardless of the value of $f$ unless $f$ is too small in which case the system dies out after just a few generations.

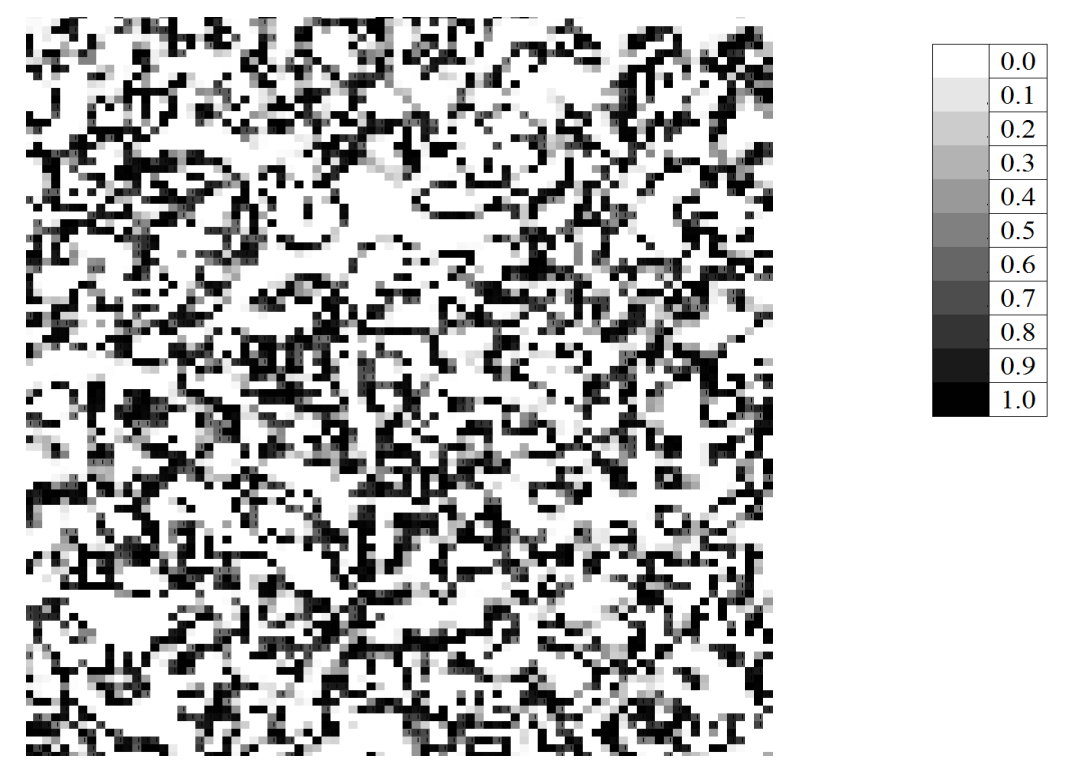

FIG. 1. The cell liveness parameter $a$ is displayed as a gray scale with values of $a$ presented in the gray-scale bar.

Even at this elementary stage there is an opportunity to develop the model. What is the minimum value of $f$ that allows a system to survive? If the system survives, the time to 
reach equilibrium depends on $f$, as shown in Fig. 2, which could be determined as a function of $f$.

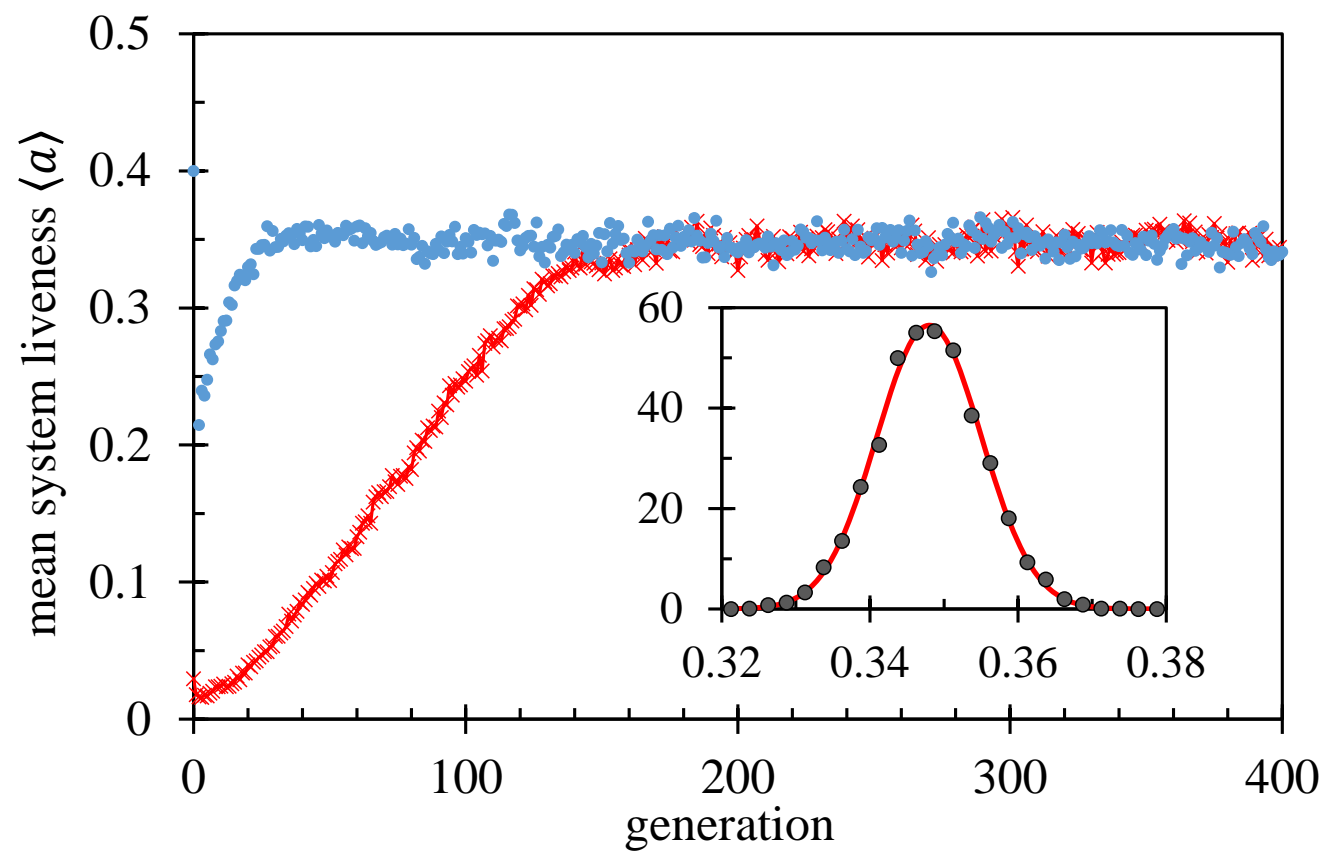

FIG. 2. The mean cell liveness $\langle a\rangle$ of a system of $100 \times 100$ cells with a starting fraction of live cells $f=0.2(\times-$ red $)$ and $f=0.8(\bullet-$ blue $)$ is presented as a function of generation. The mean cell liveness is $\langle a\rangle=0.3480 \pm 0.0001$ with a standard deviation 0.0071 as shown in the inset.

\section{B. Universal constants}

The rules of the SCGOL are dictated by two parameters: the optimum liveness $A_{\mathrm{o}}$ and a "resilience" parameter $R$. The optimum liveness is $A_{\mathrm{o}}=3$ which means that if a cell $i$ is dead a new life is produced if $A=3$. This value is chosen to match the parameters of Conway's classical game of life where the neighboring liveness $A$ is an integer. The resilience parameter $R$ defines the interval of the Moore liveness neighbourhood $A$ for which rule changes apply. For the standard rules presented in Table I, the resilience parameter $R=1$ since the rules change at intervals of 1 unit, at $A=1,2,3$ and so forth. If $R$ is reduced, individual cells will flourish for a narrower range of values of $A$ and, if $R$ is increased, individual cells will be less sensitive to local crowding or loneliness. Conceivably, creatures such as insects might be modeled by a high $R$ and large mammals by a low $R$. The present simplified game uses the same parameter set $\left(A_{\mathrm{o}}, R\right)=(3,1)$ whereupon the equilibrium liveness density distribution 
is found to have the same mean and standard deviation independent of the initial conditions as shown by the Gaussian distribution of equilibrium liveness presented as the inset in Fig. 2.

An interesting adaptation to the SCGOL would be to change the parameters $\left(A_{\mathrm{o}}, R\right)$ and explore different behaviors. New mean liveness $\langle a\rangle$ and standard deviation values would be anticipated reflecting the characteristics of the new population density. Are $A_{\mathrm{o}}$ and $R$ meaningful in any sense in terms of characterising species?

\section{Relationship to real populations}

The SCGOL provides a probabilistic description of population densities characterised by the two game parameters $A_{\mathrm{o}}$ and $R$ as an alternative to the special patterns of live cells produced by Conway's classical game and provides new possibilities for exploration. For example, the game may mimic the response of populations of creatures to a sudden reduction in population density. Suppose, at equilibrium, the liveness of each cell is multiplied by a factor $c=0.1$ and renormalized. How long does it take for the population to return to its equilibrium density or does the population die out? What is the critical value of $c$ for recovery and how does this depend on $A_{\mathrm{o}}$ and $R$ ? Such a study would attempt to relate the game to the real world through parameters $A_{\mathrm{o}}$ and $R$ where a species may be subject to a sudden population loss, perhaps due to the effect of a virus, or suffer a reduction in resilience due to environmental impact such as witnessed for corals with sea-temperature rises. More often, species may be subject to slow decline due to the loss of habitat or through hunting. This process could be simulated by setting $c \approx 0.5$ (say), renormalising, and applying the reduction at every generation.

In order to model two interacting populations, students might prepare two parallel simulations, one representing an animal such as a fox and one representing its prey, rabbits. The liveness of rabbits will diminish if surrounded by foxes and foxes will flourish if there is a high density of rabbits in the neighborhood. A simple interaction rule might allow both rabbits' and foxes' prospects to depend on a suitable weighting between the population of their own kind and of the other kind in their Moore neighborhood. Additional rules could lead to the death of a rabbit if surrounded by more than a critical number of predators and the predator could die if surrounded by too few prey, as if by starvation. How might this model compare to a standard mathematical solution using coupled differential equations, a 
Monte Carlo simulation or observations of real populations?

Unexpectedly, the SCGOL explored here may better simulate the behavior of populations than Conway's classical game for many reasons including (i) representation of populations as a probability density rather than pockets of live individuals, (ii) the ability to parameterise different species by their optimum liveness and resilience, (iii) the ability to incorporate sudden or systematic reduction in population density and (iv) the ability to run parallel games with two different species that can then interact according to additional simple rules.

\section{New lifeforms}

Students may be interested to produce a video of the evolution of liveness density. This is readily achieved if computations are executed in a package such as Matlab or, alternatively, data dumps may be read into Matlab or a similar graphical facility. Close examination of evolving systems by video will identify transient lifeforms, existing perhaps for a small number of generations before encroachment and destruction by neighboring liveness cloud. This can be seen in a video provided as supplementary information.

Some new lifeforms with part-live cells have been identified and are included in Fig. 3. A few of these have been observed directly in the simulations albeit only for a few generations before encroachment by the cloud, and others are adapted from classical lifeforms. These constitute new lifeforms and have been named empathetic to their classical equivalents where possible. Others are provided new nautical labels. The qutub and the quracle (named after the traditional Welsh wicker boat) are the most commonly-observed transient lifeforms. Each structure is presented in Fig. 3 with the range of cell liveness that provides still-lifes (or hibernating lifeforms) ${ }^{20}$, that is they are invariant to the evolutionary rules when placed in an otherwise dead universe.

Students may wish to design their own stable still-life structures. We have not found stable oscillator lifeforms with the SCGOL but Bleh ${ }^{20}$ reports a quantum blinker in a SQGOL game and oscillatory behavior has been reported elsewhere ${ }^{13,15}$. Presumably, using different evolutionary rules as suggested in Sec. III B, there will be different requirements for stable

lifeforms and possibly new lifeforms. Can any of these elementary part-live lifeforms be combined to construct larger lifeforms? 


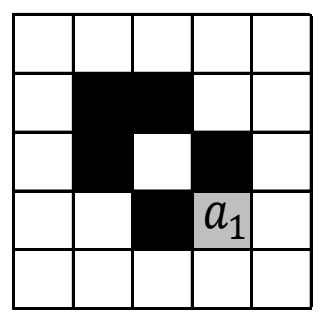

quboat
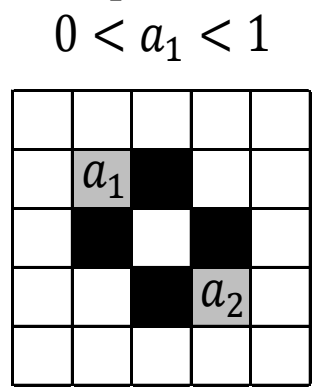

quracle
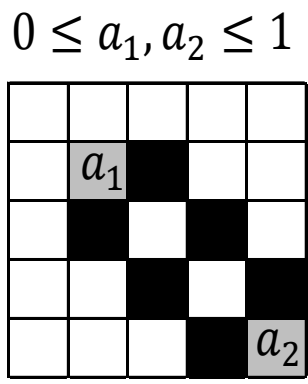

qubarge

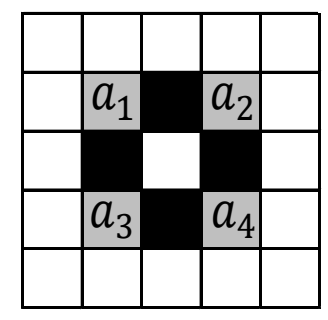

qutub
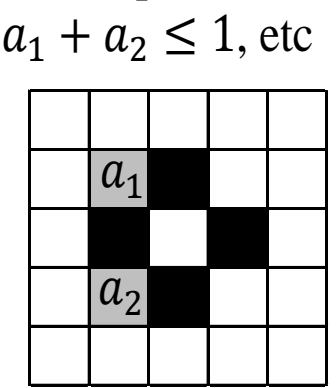

qutug

$$
a_{1}+a_{2} \leq 1
$$

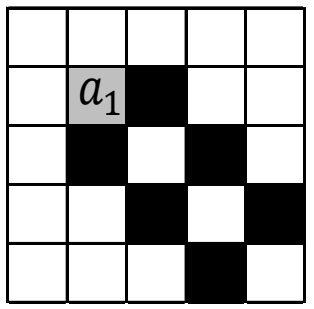

quruiser

$$
0<a_{1}<1
$$

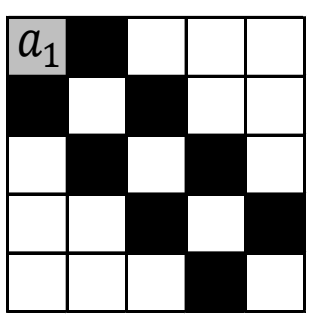

quyak

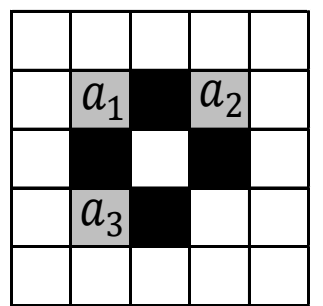

quatamaran
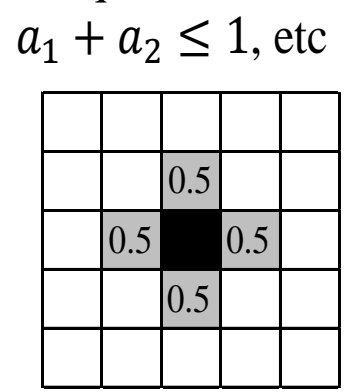

qudot

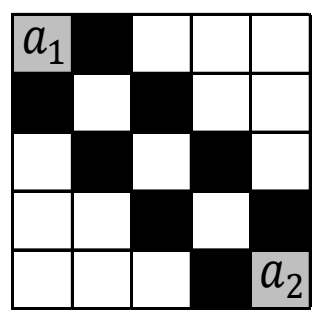

qunoe

$0 \leq a_{1}, a_{2} \leq 1$

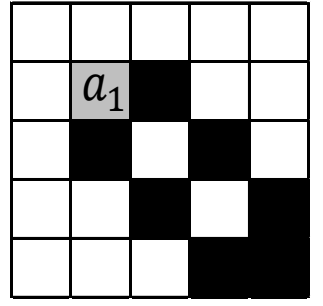

qulongboat

$$
0<a_{1}<1
$$

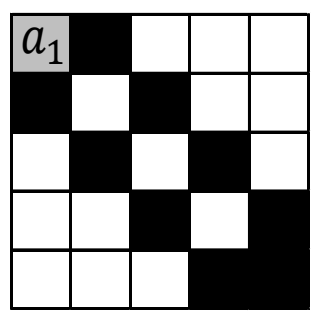

quverylongboat

$0<a_{1}<1$

FIG. 3. A selection of lifeforms possessing part-live cells are presented with the liveness criteria that ensures invariance to the evolutionary rules. The names are empathetic to their classical counterparts or created by the authors. White cells are dead, black cells are live and gray cells are part-live. 


\section{E. Seeds and floating-point error}

The qutub is a lifeform of special interest. The qutub comprises $3 \times 3$ cells of which four are live, four are part-live and the central cell is dead. The lifeform is unchanging if surrounded by dead cells and if each part-live cell satisfies the criterion that the livenesses $a_{i}$ of any pair of next-nearest (non-diagonal) neighbors sum to 1 or less. The qutub is dormant, or hibernating, until an event occurs which changes one or more of the part-live cells so that the stability criteria is no longer satisfied. Now the qutub acts as a seed to spawn a variety of structures depending on the livenesses $a_{i}$ of the four part-live cells. The hibernating qutub structure in Fig. 3 was placed at the center of an otherwise empty universe. The evolution of the qutub seed was then explored for $a_{i}=0.57,0.58$ and 0.59 for $i=1 \ldots 4$. The outcomes are presented in Fig. 4.

The evolution of the qutub seed is an example of a computer simulation with outcomes that are affected by the presence of floating-point error. The loss of the four-fold symmetry in the case of the qutub seed with $a_{i}=0.58$ is due to floating-point precision limits due to the mapping of real numbers to a finite number of bits. For example, executing the Moore neighborhood summation in the order $a_{1}+a_{2}+a_{3}+a_{4}+a_{5}+a_{6}+a_{7}+a_{8}$ can yield a very slightly different result compared to $a_{5}+a_{6}+a_{7}+a_{8}+a_{1}+a_{2}+a_{3}+a_{4}$ because of the order in which pairs of additions are taken. This can be seen clearly for the example summarised in a spreadsheet provided as supplementary material for an initial qutub seed with all part-live cells initiated to 0.50006. All numerical values of liveness are presented every 10 generations to the double precision limit of 15 digits. After 10 generations, the four-fold symmetry is retained to 15 digits. After 20 generations, there are eight cells which should have precisely the same liveness if the four-fold symmetry is retained but in fact have a range that differ by five at the 15th decimal place. Once a small difference has occurred, the variation increases as the number of generations increases, with differences at the 12th, 11th, eighth and fifth decimal places at generations 30,40,50 and 60 respectively. The calculation of the liveness of a Moore neighbourhood might now fall either side of a rule boundary and the evolution of each cell is therefore slightly different. By generation 70, differences are at the second decimal place and by 80 generations the four-fold symmetry is clearly lost.

Floating-point errors are not random errors, they are systematic errors. Exactly the same difference will occur each time the code is executed on the same computer and therefore 


\begin{tabular}{|c|c|c|}
\hline$a_{i}=0.57$ & $a_{i}=0.58$ & $a_{i}=0.59$ \\
\hline $\begin{array}{r}n=0 \\
\\
\\
\quad \text { a }\end{array}$ & $n=0$ & $\begin{array}{l}n=0 \\
\\
\end{array}$ \\
\hline $\begin{array}{l}n=16 \\
\text { 些 }\end{array}$ & $\begin{array}{l}n=32 \\
\quad 00\end{array}$ & 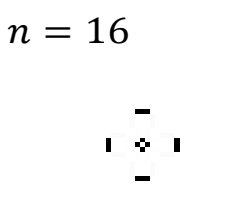 \\
\hline $\begin{array}{c}n=32 \\
-\quad-\end{array}$ & $\begin{array}{l}n=64 \\
\end{array}$ & $n=32$ \\
\hline$n=48$ & $\begin{array}{l}n=96 \\
\\
+59\end{array}$ & 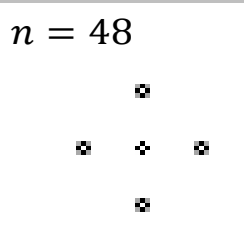 \\
\hline$n=64$ & 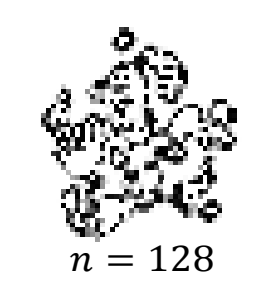 & 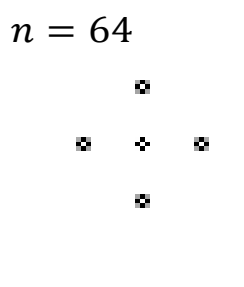 \\
\hline
\end{tabular}

FIG. 4. The evolution of a qutub seed with $a_{1}=a_{2}=a_{3}=a_{4}$ equal to the values shown at generation $n$ are presented. White cells are dead, black cells are live and gray cells are part-live. The figure illustrates the variety of final outcome for small differences in the liveness of the part-live cells. The extended number of generations in the central column serves to illustrate that the cloud expands to eventually fill the system space. Videos of each example are provided as supplementary material.

exactly the same simulation will arise for the same initial conditions. Game evolutions might therefore be slightly different when executed using single precision or on different CPUs. The consequence in the simulations presented in Fig. 4 is that the evolution to a cloud rather than a system with four-fold symmetry is due to the escalation of floating-point errors. Videos of 
each evolution in Fig. 4 are provided as supplementary material. If $a_{i}=0.59$, the evolution produces a central tub plus four satellite qutubs. Thus, the qutub reproduces to provide four new still-life child qutubs. If the qutub seed has its four part-live cells initiated at 0.57 , the system evolves but dies out after about 40 generations. Both outcomes are achieved before the full impact of the floating-point errors are felt. By contrast, for $a_{i}=0.58$, the qutub seed initially evolves with four-fold symmetry as expected but the symmetry is clearly lost after about 60 generations with the subsequent evolution producing a space-filling liveness cloud as illustrated in Fig. 4.

We consider the propagation of floating-point errors in the qutub simulations leading to the cloud as an appealing feature of the simulation in that the outcome appears to be statistically indistinguishable from the stock simulation that evolves from a random initial starting configuration. If the SCGOL purports to mimic biological systems, then imperfections would be present such as seen in the growth of bacteria or mould. Nonetheless, it would be interesting to explore the evolution of the qutub seed while eliminating, or significantly reducing, the impact of floating-point errors.

In principle, the elimination of floating-point errors could be achieved in the case of the four-fold-symmetric qutub seed simulation by replacing a numerical result that differs from its three identical images by its image number. The procedure relies on just one of the four symmetric numbers differing in an iteration and assumes that this single outlier is the incorrect number, rather than the remaining three. Furthermore, some programming languages have built-in support or supply libraries for arbitrary-precision arithmetic (for example for the $\mathrm{C}++$ programming language ${ }^{24}$ ) which could be used to explore near errorfree SCGOL evolution. This would prove an interesting avenue for research.

More interesting in the present context is the recent revelation that the escalation of floating-point error might contribute to the statistical properties of chaotic dynamic systems $^{25}$. It is conceivable that digital representation of floating-point numbers provides an additional, possibly significant, contribution to those properties obtained from statistically-averaged simulation data ${ }^{26}$. We tested the possibility for a SCGOL simulation on a $500 \times 500$ grid with an initial random configuration of liveness which was allowed to evolve for 30 generations with the final liveness configuration written to file to just six decimal places. The simulation is too short to allow the propagation of floating-point errors to appear in the first six decimal places (having progressed to about the 11th decimal 
place as seen in the supplementary material). This final configuration was then read into a fresh simulation which was run for 130 generations. The mean and standard deviation of the liveness was determined for generations 10 to 40 inclusive and for generations 100 to 130 inclusive. The first sample is too short for floating-point errors to have impacted simulation and the second is long enough for floating-point errors to have dominated the arithmetic based on the evidence from the evolution of qutub seeds. The former provided $\langle a\rangle=0.3464 \pm 0.0020$ and the second $\langle a\rangle=0.3482 \pm 0.0013$ confirming that floating-point error does not impact these specific statistics in this game.

\section{F. Chaos-like behavior}

In this study, the liveness of the diagonal part-live cells of the qutub seed were set equal, that is $a_{1}=a_{4}$ and $a_{2}=a_{3}$. The evolution of the qutub seed was then explored for $a_{1}=a_{4}=0.5 \ldots 1.0$ and $a_{2}=a_{3}=0.5 \ldots 1.0$ at intervals of 0.01 for 400 generations. Evolutionary outcomes are presented in Fig. 5. With certain parameters, the qutub evolves with two- or four-fold symmetry and then dies out. With other parameters, one or more classical or part-live lifeforms evolve to form new qutubs, quracles or combinations of these. A wide variety of lifeform structures can emerge. One case leads to 16 classical blinkers, others to the classical tub or combinations of classical and part-live lifeforms. Occasionally a structure is stable for more than four generations before evolving to cloud or death. Where discovered, these are indicated as half squares in Fig. 5.

If the dead universe or stable lifeforms do not arise within 60-80 generations, the liveness cloud emerges such as that illustrated in Fig. 1. In these simulations, floating-point errors were not corrected and so the emergence of a system of static lifeforms such as those illustrated in Fig. 4 must arise prior to 60-80 generations before floating-point errors dominate the evolution. The cloud would not arise in a simulation that corrected for floating-point error and the two- or four-fold symmetry would presumably be retained. One may speculate that outcomes such as death, static lifeforms or even oscillators may emerge at later generations. This is left as an opportunity for exploration. The objective here is to demonstrate the sensitivity of the evolution to the initial conditions which will pervade whether floating-point error is corrected or not.

In the SCGOL, the small numerical differences arising due to floating-point precision 


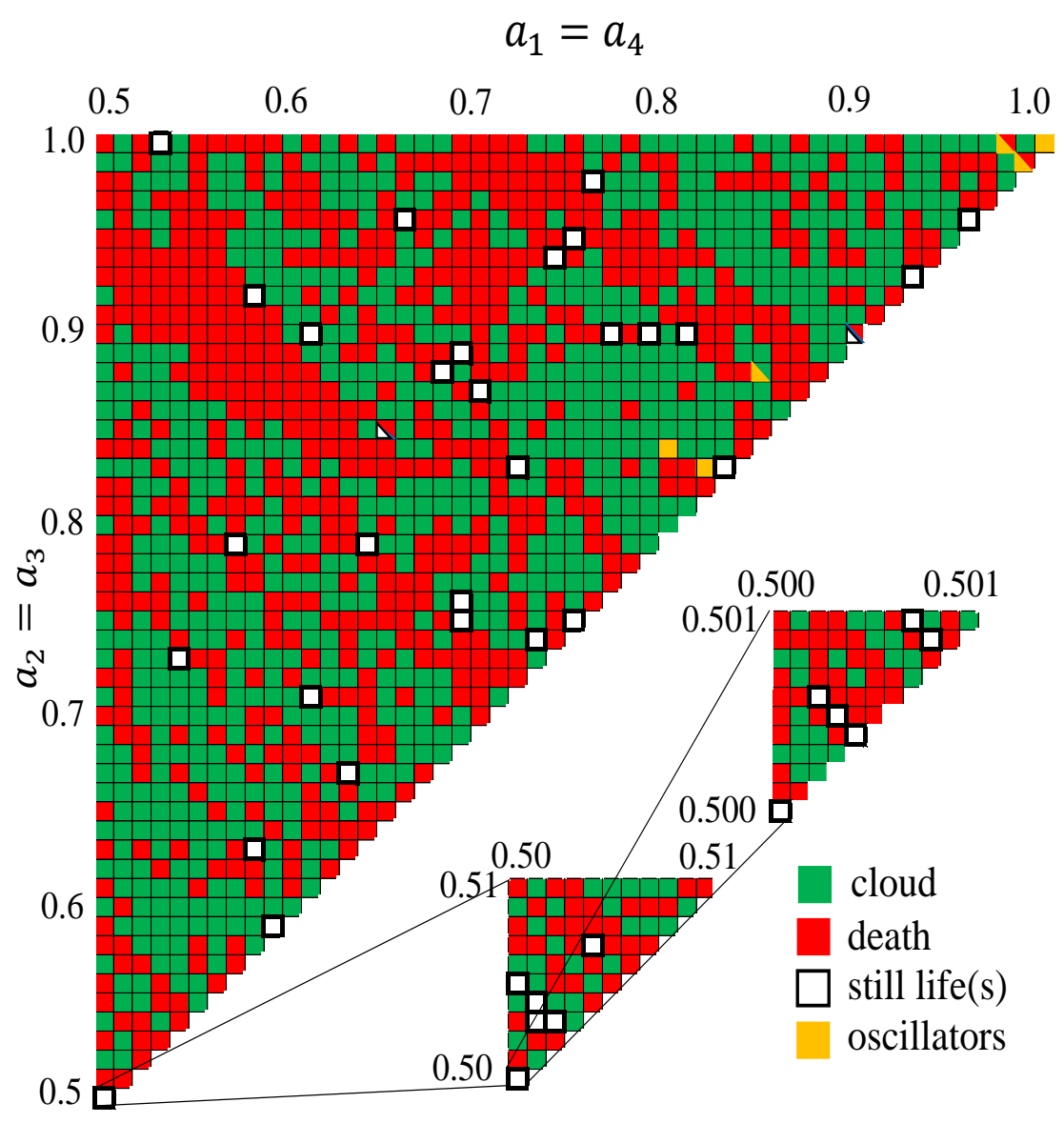

FIG. 5. The evolutionary outcomes of a qutub seed with initial livenesses $a_{i}$ are presented. Halfcells indicate temporary forms.

limits could be considered as the butterfly wings that lead to a chaos-like behavior. A chaotic behavior in dynamic systems may be characterised by the Lyapunov exponent. A small difference in initial conditions leads to a divergence of outcome that can be described by an exponential function characterised by the Lyapunov exponent. This is not possible here due to the variety of possible outcomes of the game for tiny differences in initial conditions. For example, if all four part-live cells of the seed qutub are set to 0.50006, the cloud is produced. If all four part-live cells are set to 0.50007, the system dies out, and for 0.50008 the final outcome is a single new qutub with the four part-live cells equal to 0.0714 .

The variety of outcomes and sensitivity to the initial conditions for the evolution of the qutub seed is illustrated in Fig. 5 for the full parameter space. The diagonal line of results in Fig. 5 correspond to initial state $a_{1}=a_{2}=a_{3}=a_{4}$. If $a_{1}=a_{2}=a_{3}=a_{4}=0.5$, the qutub is a still-life (unchanging). This is represented by the open square at bottom left in 
Fig. 5. An exploration of the part-live parameter space at finer divisions of 0.001 for the range $0.50 . .0 .51$ and divisions of 0.0001 for the range 0.500..0.501 are shown in Fig. 5. These yield a distribution of cloud, death and still-life outcomes. A similar sensitivity of outcome to the initial conditions is therefore observed at different scales.

We have only explored the evolution of a qutub seed with the restriction $a_{1}=a_{4}$ and $a_{2}=a_{3}$. Other combinations provide rich territory for exploration. The evolution of other structures in Fig. 5 when the stability criterion is breached has not been explored. Does the phenomena presented in Fig. 5 constitute scaling behavior?

A simulation in which a liveness cloud emerges from a seed qualitatively represents evolution of viruses or bacteria from an initial source as a population density. A simple modification of the rules so that each cell dies once its supply of food has been exhausted, that is once the cumulative liveness over a number of generations exceeds the critical limit. Such a simulation might mimic the evolution of moulds as one might see in the production of blue cheeses.

\section{G. The semi-quantum game of life}

A variety of new part-live lifeforms have been presented in Fig. 3. These are all still-lifes. We have not, however, discovered any new lifeforms with the SCGOL that are oscillators, though we believe they exist. All oscillators that emerge from our game as indicated in Fig. 5 are classical oscillators containing only dead and alive cells and are the same as those seen in Conway's classical game. However, oscillatory behavior has been observed in simplified versions of the full SQGOL implementation described in Sec. II ${ }^{13,16,20}$. The implementation of the full SQGOL is achieved by introducing complex initial livenesses and setting $\phi \neq 0$, then oscillatory behavior between neighbouring cells will become apparent ${ }^{13,15,20}$. It would be particularly interesting to study the evolution of the qutub with rules of the SQGOL.

\section{SUMMARY}

Cellular automata are widely used in undergraduate physics courses as effective means of developing students' programming skills and for project work where complex phenomena can be explored on the basis of simple algorithmic rules. The cellular automata, here illus- 
trated through a classical game based on the rules of the semi-quantum game of life, adds a new dimension to project work. The protocol for the SCGOL is easily implemented using standard programming packages. The SCGOL spawns an extraordinary range of differing phenomena, most of which are not seen in Conway's classical game, and which provide fertile ground for further exploration by students in project work. Numerous ideas for extending the simplified game are suggested and the trivial extension to the semi-quantum game where quantum effects may be seen is presented.

The results presented here represent the sum total of work with the SCGOL. This game provides numerous possibilities for modification compared to Conway's classical counterpart, allows undergraduate students to explore new research territories, and to develop their own ideas as, indeed, did two of the authors.

* d.faux@surrey.ac.uk

1 J. Von Neumann, Math. Ann. 100, 295 (1928).

2 J. Von Neumann and O. Morgenstern, Game theory and economic behavior (Princeton, NJ: Princeton University Press, 1944).

3 M. Gardner, Sci. Am. 223, 120-123 (1970).

4 C. Hauert and G. Szabó, Am. J. Phys. 73, 405 (2005).

5 D. Challet and Y.-C. Zhang, Physica A 256, 514 (1998).

6 G. Faraco, P. Pantano, and R. Servidio, Comput. Educ. 47, 280 (2006).

7 A. Dhatsuwan and M. Precharattana, Simulat. Gaming 47, 445 (2016).

8 J. Ogborn, J. Comput. Assist. Lear. 6, 103 (1990).

9 S. Wolfram, Physica D: Nonlinear Phenomena 10, 1 (1984).

10 Wikipedia, "Conway's Game of Life," (2019), last accessed: 2019-02-21.

11 R. P. Feynman, Int. J. Theor. Phys. 21, 467 (1982).

12 J. Eisert, M. Wilkens, and M. Lewenstein, Phys. Rev. Lett. 83, 3077 (1999).

13 A. P. Flitney and D. Abbott, "A semi-quantum version of the game of life," (2002), arXiv:quant$\mathrm{ph} / 0208149$.

14 A. López-Incera and W. Dür, Am. J. Phys. 87, 95 (2019).

15 A. P. Flitney and D. Abbott, in Advances in Dynamic Games (Springer, 2005) pp. 667-679. 
16 A. P. Flitney and D. Abbott, in Quantum Aspects of Life, edited by D. Abbott, P. C. Davies, and A. K. Pati (World Scientific, 2008) pp. 233-250.

17 G. S. Engel, T. R. Calhoun, E. L. Read, T.-K. Ahn, T. Mančal, Y.-C. Cheng, R. E. Blankenship, and G. R. Fleming, Nature 446, 782 (2007).

18 M. Mohseni, P. Rebentrost, S. Lloyd, and A. Aspuru-Guzik, J. Chem. Phys. 129, 11B603 (2008).

19 D. Abbott, P. C. Davies, and A. K. Pati, Quantum Aspects of Life (World Scientific, 2008).

20 D. Bleh, T. Calarco, and S. Montangero, Europhys. Lett. 97, 20012 (2012).

21 F. Bagarello, R. Di Salvo, F. Gargano, and F. Oliveri, Appl. Math. Model. 43, 15 (2017).

22 P. Arrighi and J. Grattage, Physics World 25, 23 (2012).

23 T. Ceccherini-Silberstein and M. Coornaert, Cellular Automata and Groups (Springer Berlin, 2013).

24 "Boost releases on github," (accessed 5 December 2019), https://github.com/boostorg/boost/releases.

25 B. M. Boghosian, P. V. Coveney, and H. Wang, Advanced Theory and Simulations 2, 1900125 (2019).

26 The authors ${ }^{25}$ illustrate error-some calculations using the Bernoulli map $x_{i+1}=\bmod \left(\beta x_{i}, 1\right)$ with $\beta=2$. The repeating sequence $0.6,0.2,0.4,0.8,0.6 \ldots$ has $\langle x\rangle=0.5$ but an eight-line code in MATLAB yields $\langle x\rangle \rightarrow 0.0$ when executed for a large number of iterations. 


\section{Figure captions}

FIG 1. The cell liveness parameter $a$ is displayed as a gray scale with values of $a$ presented in the gray-scale bar.

FIG 2. The mean cell liveness $<a>$ of a system of $100 \times 100$ cells with a starting fraction of live cells $f=0.2$ (red) and $f=0.8$ (blue) is presented as a function of generation. The mean cell liveness is $\langle a\rangle=0.3480 \pm 0.0001$ with a standard deviation 0.0071 as shown in the inset.

FIG 3. A selection of lifeforms possessing part-live cells are presented with the liveness criteria that ensures invariance to the evolutionary rules. The names are empathetic to their classical counterparts or created by the authors. White cells are dead, black cells are live and gray cells are part-live.

FIG 4. The evolution of a qutub seed with $a_{1}=a_{2}=a_{3}=a_{4}$ equal to the values shown at generation $n$ are presented. White cells are dead, black cells are live and gray cells are part-live. The figure illustrates the variety of final outcome for small differences in the liveness of the part-live cells. The extended number of generations in the central column

serves to illustrate that the cloud expands to eventually fill the system space. Videos of each example are provided as supplementary material.

FIG. 5. The evolutionary outcomes of a qutub seed with initial livenesses $a_{i}$ are presented. Half-cells indicate temporary forms. 